\title{
Under the Activities-based Costing method of the construction of environmental cost accounting
}

\author{
Wang Wanji ${ }^{1, a,{ }^{*}}$,Tang Wangwei ${ }^{2, b}$ \\ ${ }^{1}$ School of Management,Wuhan University of Technology, Wuhan ,Hubei,China \\ ${ }^{2}$ School of Economics, Yangtze University,Jingzhou, Hubei,China \\ aemail:School8855@163.com, bemail: angwanwei8855@163.com
}

${ }^{*}$ Corresponding author

Keywords: Environmental Cost; Accounting Recognition; Measurement; Cost Control

\begin{abstract}
The current problems of environmental cost accounting treatment are not conducive to the commitment of environmental responsibility, but also detrimental to the construction of "environment-friendly society".Institutional barriers to environmental cost accounting will also hamper the effectiveness and sustainability of the business. This paper analyzes the problems existing in the aspects of confirmation, measurement, disclosure and accounting of environmental costs due to the lack of laws and regulations, and further puts forward some suggestions for improvement based on the problems. At the same time, this paper uses the Activity-based Costing method to optimize the existing accounting methods according to the production links. Through this method can accurately account for all aspects of the corresponding environmental costs, clear the responsibility of the independent departments of the cost, is conducive to enterprise environmental cost control.
\end{abstract}

\section{作业成本法下企业环境成本的核算机制的构建}

\author{
王万吉 $1, \mathrm{a},{ }^{*}$ ，唐王维 ${ }^{2, \mathrm{~b}}$ \\ 1 武汉理工大学管理学院, 武汉, 湖北, 中国 \\ 2 长江大学经济学院, 荆州, 湖北, 中国 \\ aemail:School8855@163.com, bemail : angwanwei8855@163.com \\ 通讯作者
}

关键词: 环境成本; 会计确认; 计量; 成本控制

中文摘要. 当下环境成本会计处理方面的各种问题不利于企业环境责任的承担, 更不利于

“环境友好型的社会” 建设。环境成本会计不足带来的制度障碍也将对企业的效益提升及可 持续发展构成阻碍。针对目前环境成本在确认、计量、披露及核算等各环节因缺少法律法规 的指导而存在的各种问题本文首先进行了分析, 进一步根据问题提出了相关的改善建议。同 时, 本文创新的利用作业成本法结合企业各个生产环节, 对现有的核算方法进行优化。通过 该方法能够准确核算企业各个环节对应的环境成本, 明确各独立部门的成本责任, 有利于企 业环境成本控制。

\section{1. 引言}

环境成本又称环境降级成本，概念首次涉及是1993年联合国统计司在 “综合环境与经济 核算体系” 的初稿当中提出的。该体系概况环境成本有两方面组成: 一方面, 企业在各种环 境保护方面的实际支出; 另一方面, 自然资源的数量减少和质量的下降导致的资源价值减 
少。该概念的明确, 不仅体现了人类对环保的重视、也是对企业履行各种环保义务的硬性要 求。

中国经济过度依赖自然资源在取得了良好的增长的同时也带来不小的环境问题。资源过 度消耗及环境恶化引起了政府的重视, 提出并明确要求全面建设 “环境友好型的社会”。政 府及社会各界对环保问题的不断重视最终传导至企业在环保等方面的各种重视，节能减排成 本、排污成本都是企业不得不面临的问题。正是如此, 环保理念深化及节能减排的硬性要求 不断提高, 导致企业环境成本不断增加。因此, 将与环境相关的直接成本、间接成本纳入企 业财务核算体系，充分发挥企业会计在环保中的核算与监督职能 ${ }^{[1]}$ 。

\section{2 环境成本核算制度历史介绍}

\section{1 国外相关规定}

首先，美国财务会计准则委员（FASB）会在全球首个尝试性的提出与环境成本核算相关 的制度。1989年在其 “EITF89-13（FASB旗下的紧急问题特别委员会）一一关于石棉清理成 本” 的公告中明确提及环境成本核算。紧随其后，1990在FASB在 “EITF90-8一一环境成本资 本化” 的公告中进一步对环境成本的资本化进行了更严格的标准化。2001年FASB发布了 “FAS143号文一资产弃置义务会计核算”，该文涉及环境成本并对与环境资产相关的弃置进 行了说明, 要求企业环境资产弃置成本在初始确认时计入相关长期资产（资本化处理）, 并 在后续按照规定对其进行摊销。

1993年，加拿大特许会计师协会（CICA）在其公布的 “环境成本负债的会计核算与报 告” 发文中明确了环境成本概念并将其分为环境措施成本即环境损失成本两类。要求企业对 现在的环境成本及未来潜在的环境支出都需在当期进行会计核算, 该文也对环境成本的资本 化及费用化进行了界定，还对与此相关的列报披露问题有所交代。随后，CICA在当年的加拿 大环境调查报告中详细的介绍了1993年加拿大 863 家企业在环境成本与负债等方面的会计核 算情况。

1998年，“联合国国际会计和报告标准政府间专家工作组”（UNCTDA）在当年的第15次 会议上正式通过并对外公布了 “环境会计和报告的立场公告”，此公告成为第一份关于环境 成本会计与报告的正式性的国际指南。该公告对环境成本的定义, 确认、计量和报告披露均 系统性论述的基础上给出了说明。首先, 环境成本定义。环境成本是指：1. 管理企业环境问 题采取行动而发生的成本支出；2. 企业为实现预期的环境目标发生的其他支出。其次，环境 成本确认、计量及披露。报告要求企业在初次识别环境成本的会计期间确认并根据该成本支 出的未来经济利益流入性能区分资本化与费用化。最后，报告要求企业按照环境成本的具体 类别, 严格按照重要性原则考虑分别在财报及附注中列报、披露, 必要时考虑利用专项报告 对其披露。不过该报告美中不足，仅涉及到企业内部的环境成本，并未对企业外部的各种环 境成本进行讨论并说明。

\section{2 国内相关规定}

除了上世纪九十年代初部分学者开始将各种国际上有关的环境成本在理论上引入国内 外, 国内企业会计准则或会计制度尚未专门针对环境成本的核算进行规范化的说明或规定。 不过，现行的会计法律体系中也逐渐涉及到各种环境成本的核算。2014年的企业会计准则解 释第6号指出企业需要考虑预计固定资产的弃置费用等因素对固定资产初始确认成本的影 响; 更进一步说明弃置义务发生时导致预计负债变动, 需针对具体情况是否应调整固定资产 的初始确认成本。

同样，最新2014年修订的“CAS5-生物资产”有所涉及，该准则规定企业用于环保目的 的生物性资产为公益性的生物资产并对其有关核算进行简单的说明。CAS5号文规定企业按照 郁闭前的各种直接费用和待分推的间接费用确认自造公益生物资产并计量 ${ }^{[2]}$ 。 


\section{3 当前对此核算的潜在问题}

虽然国际上已经出现很多关于很多环境成本核算的指导性文件以及众多的理论研究，也 为环境成本会计核算构建了一定的框架体系; 但是会计准则角度来看, 仍旧缺乏标准化、规 范化的规定与此相对应, 尤其在国内。会计法律制度上的不足及缺少为企业在环境成本方面 的核算带来了众多的问题, 也导致企业配套的管理跟不上, 拖累我国 “环境友好型的社会” 的建设。

\section{1 确认}

国际、国内实务中，企业环境成本确认众多不一，方式方法缺少标准。目前对环境成本 予以会计上的确认问题，企业基本一致认可; 不过，企业在生产经营过程中各种环境成本确 认的资本化和费用化分歧颇大。目前国际主流的环境成本资本化观点就有两种，第一种观点 以 “经济利益的流入” 为认定标准, 只有环境成本发生后能为企业带来经济利益才可资本化 确认，反之费用化; 第二种观点以 “利益相关论” 为认定标准, 无论是否能带来经济利益流 入, 只要环境支出能对企业的存续带来好处就需资本化处理, 反之费用化。国内相关观点基 本在这两观点的基础上衍生而得, 此处不在重复。

针对两者观点，可以发现第二种 “利益相关论” 比 “经济利益流入” 观点涵盖的范围稍 广, 解决二者的分歧就在于两者的不同。两者不同点：主要针对的是 “各种环境相关支出不 能带来经济利益流入却对企业持续生存有利”。因此, 对此点分歧解决将可以有效环境目前 实务及理论中对环境成本确认资本化及费用化的分歧。

\section{2 计量}

因环境成本支出的复杂性及其自身特殊性带来了计量上存在很多问题、甚至缺陷，具体 可以从内部环境成本与外部环境成本来分类讨论。

针对内部环境成本的计量问题，目前问题较小，主要就是实务中企业采取的计量方法众 多不统一, 还有就是每种计量方法所适用的具体项目不统一。缺乏统一的会计准则指导, 导 致计量差异, 最终形成的环境成本信息在不同企业之间失去了可比性, 也可能违背重要性原 则。

目前问题严重的是企业外部环境成本问题, 主要存在两点问题。一是企业考虑了外部环 境成本但是难以估计计量。外部环境污染或破坏带来的损失支出等虽不需企业承担支出, 但 是由企业生产经营所导致, 所以很多企业考虑了该成本, 不过货币计量此时成为了难点, 目 前缺少专业的计量技术。二是一些企业直接忽略外部环境成本。考虑外部环境成本终究无需 企业支付导致部分企业直接忽视不予以考虑（更不说计量）。不过, 环境污染和破坏对社会 及人类产生的危害又确实存在, 核算的重要性不可忽视。因此, 目前计量的方法及是否计量 都是问题。

\section{3 披露}

笔者整理2011年-2015年国内上市公司社会责任报告中各种与环境成本相关信息的披露 情况发现, 随着政府和社会对环境问题的不断重视, 企业在环境方面的重视程度也逐年增 加。在笔者的统计中国可发现, 自11年至15年上市公司对与环境成本相关的各种信息披露数 量逐年增加。

不过，重视程度总体来说依然不高有待提高，以2015年为例，上市公司披露此方面信息 的家数共有 812 家仅占上市公司总数的三分之一左右。除此之外, 环境成本信息在上市公司 的披露中整体质量不高、可比性不足、准确性更有待商榷等问题依然较为突出。目前国内上 市公司大多数喜欢避重就轻、报喜不报忧的披露各种好的一面, 坏的一面往往只字不提; 还 
有众多上市公司披露方式方法严重不统一，缺乏行业标准或国际标准，没有可比性; 最严重 的可能还是信息的可靠性问题，大量的信息缺乏有效的会计数据信息支持，更没有专业机构 的鉴定报告，导致准确性偏低。

总的来说，缺少法律法规的硬性标准化的规定，导致国内企业披露的各种环境成本信息 可使用性和价值性都大打折扣。

\section{4 核算方法}

目前国内企业在对环境成本的核算过程中大多数采用的是完全成本法，虽然较为统一但 过度单一的方法并不能充分适应于企业的经营管理，尤其成本核算与控制。完全成本法的最 大优点是对企业环境成本的综合性及全面性; 不过过度综合导致该方法没有有效区分核算 内、外部环境成本，影响环境成本核算的针对性和准确性。

除此之外，目前绝大多数企业只将与生产环节的相关的环境成本作为核算对象，并未将 产品设计、物资采购、产品销售等环节相关环境成本纳入核算体系，导致环境成本核算无法 实现社会及政府预期的目标，更不能满足企业可持续发展的要求 ${ }^{[3]}$ 。

\section{4 改善环境成本核算的若干建议}

\section{1 确认问题的改善}

企业与环境有关的各种支出采取规范化的标准认定并利用支出收益期间原则判定资本化 还是费用化是确认的关键所在。笔者认为, 企业应利遵循时间维度在各个生产经营环境对此 认定并进一步以 “是否了能在未来带来经济利益净流入” 为标准确认资本化或费用化的环境 成本。

例如，企业产品或服务的研发设计环节发生的各种与环境相关的支出，因该项支出会创 新企业产品或服务或改进企业生产技术，预期未来会给企业带来经济利益流入，故此类环境 支出应予以确认为资本化的环境成本。

再例如，企业生产经营过程中因环保各种问题不达标导致的行政罚款，此类费用明显不 会为企业带来未来的经济利益流入, 故应该在发生时立即确认为当期的费用, 费用化环境成 本。

具体企业生产经营不同环节的环境成本确认问题见下表1:

表1 生命周期法下环境成本的确认

\begin{tabular}{c|l|l}
\hline \multirow{2}{*}{ 生命周期各阶段 } & \multicolumn{1}{|c}{ 环境成本确认内容 } & 资金化/费用化 \\
\hline \multirow{2}{*}{ 产品设计 } & 环保技术研发费用 & 资本化 \\
\cline { 2 - 3 } & 预提环保准备金 & 费用化 \\
\hline \multirow{2}{*}{ 原材料采购 } & 环保材料的购成本 & 费用化 \\
\cline { 2 - 3 } & 材料运输造成的大气污染 & 费用化 \\
\cline { 2 - 3 } & 原材料消耗的自然资源 & 费用化 \\
\hline \multirow{2}{*}{ 产品生产 } & 环保设备的购置 & 费用化 \\
\cline { 2 - 3 } & 环保设备的改造、维护、折旧等相关费用 & 费用化 \\
\hline
\end{tabular}




\begin{tabular}{l|l|l}
\hline & 环保人员的相关工资费用 & 费用化 \\
\cline { 2 - 3 } & 污染物排放费用 & 费用化 \\
\cline { 2 - 3 } & 污染物排放造成的环境污染 & 费用化 \\
\cline { 2 - 3 } & $\begin{array}{l}\text { 污染物排放超标等过失行为引起的罚款、罚 } \\
\text { 金甚至赔偿 }\end{array}$ & 用化 \\
\hline \multirow{2}{*}{ 产品销售 } & 产品包装物成本 & 费用化 \\
\cline { 2 - 3 } & 运输造成的大气污染 & 费用化 \\
\hline \multirow{2}{*}{ 产品回收 } & 回收工艺的支出 & 费用化 \\
\cline { 2 - 3 } & 废弃物处理成本 & 费用化 \\
\cline { 2 - 3 } & 废弃物回收及再利用成本 & 费用化 \\
\hline
\end{tabular}

\section{2 计量方法改进}

内部环境成本的计量。目前内部环境计量准确性并没有太大问题, 关键问题是在计量过 程中经常出现计量方法与适用情况的不匹配。为此笔者针对常用的三种计量方法与此具体适 用的情形进行了配对，见下表2:

表2 内部环境成本计量方法及其适应情况

\begin{tabular}{|c|c|c|c|}
\hline $\begin{array}{c}\text { 环境成本分 } \\
\text { 类 }\end{array}$ & 计量方法 & 说明 & 适应情况 \\
\hline \multirow[t]{3}{*}{$\begin{array}{l}\text { 内部环境成 } \\
\text { 本 }\end{array}$} & 金额计量法 & $\begin{array}{l}\text { 将一个会计期间与环境有关的支出全部计入 } \\
\text { 环境成本。优点是简单易行, 针对性强, 缺 } \\
\text { 点是可靠性和准确性较低 }\end{array}$ & $\begin{array}{l}\text { 环保专设机构、 } \\
\text { 环境体系的构 } \\
\text { 建、环保技术的 } \\
\text { 研发 }\end{array}$ \\
\hline & 差额计量法 & $\begin{array}{l}\text { 用指出的额全部金额除去没有环保作用的那 } \\
\text { 部分金额计入环保成本, 有利于按功能进行 } \\
\text { 成本的划分 }\end{array}$ & $\begin{array}{l}\text { 环保资产的投 } \\
\text { 资，环保材料的 } \\
\text { 采购 }\end{array}$ \\
\hline & $\begin{array}{l}\text { 比例分配计 } \\
\text { 量法 }\end{array}$ & $\begin{array}{l}\text { 根据一定比例将环境成本分配进入产品成 } \\
\text { 本, 针对经营成本和环境成本划分不明确的 } \\
\text { 情况 }\end{array}$ & $\begin{array}{l}\text { 生产车间的污染 } \\
\text { 物处理 }\end{array}$ \\
\hline
\end{tabular}

外部环境成本的计量。外部环境成本被大多数企业忽视最关键的原因并不是与企业无 关，核心的问题的该项成本的金额难以准确确认、即便估值难度也较大。针对外部环境成本 无法准确及全面的价值确认，行业通用的计量方法大多以估计为原则。常见方法有以下四 种, 建议企业可针对不同的外部环境成本类型谨慎的选择针对性的计量方法。 
方法一，俗称 “直接计量法”，该方法适用和针对某些有确切金额的各种环境损失成 本。比如企业各种环境污染导致的行政罚款、生产率变动支出; 再比如企业环保方面采取的 各种预防性的费用等。

方法二，“间接计量法”，针对各种不确定金额的环境成本进行计量，主要指企业对维 持环境原有水平需要花费的支出对环境损失进行估计。

方法三，“替代市场法” , 针对各种不确定金额的环境成本进行计量。企业经常通过各 种替代的方法估计环境成本，比如环境成本用环境恶化带来的资产价格下跌来计量。

方法四, “意愿调查法” , 针对各种不确定金额的环境成本进行计量。企业通过询问相 关专业人员在环境改善各种措施过程中愿意支付的价格来进行估计并计量。

\section{3 与环境成本相关的各种信息披露改善}

环境成本相关的信息列报披露的改善主要取决于政府对企业的监管硬性措施的强化和企 业自身的环保意识、环境责任的提高。目前来看, 绝对主导地位的依然是政府, 为此笔者建 议政府从监管层面强化企业对各种环境成本信息的列报与披露。政府及相关部门需尽快制定 或完善与各种环境成本核算（包括信息披露）相关的规范性的制度文件，在制度约束的同时 也许积极鼓励以国企和上市公司为代表的企业主动、及时、全面的披露环境信息，带动全社 会各企业在环境信息披露方面往良好的方向发展。除此之外，还需要政府在企业在环保责任 履约、环境信息披露等方面予以重点关注, 要求企业充分披露其在环境责任重的履约情况以 及各种负面的环境信息。最后, 政府最好能够要求企业进一步披露与环境信息相关的计量方 法及数据来源, 并可以考虑让企业环境信息方面的报告经第三方专业机构审阅或鉴证, 以提 高信息质量。

\section{3 核算方法的建立健全}

针对核算方法的过度笼统不能满足企业生产管理或成本核算控制的要求，笔者建议利用 作业成本法结合企业各个生产环节, 对核算方法进行优化。企业可以将生产每一个环节发生 的（间接或直接的）环境成本预先归集为专项环境成本库, 然后在根据成本动因（作业法的 两大成本归集动因之一）对成本进行分配计算。

企业生产经营不同环节的环境成本动因可能差异较大。比如，设计环节企业考虑的低碳 环保理念融入产品设计; 材料采购环境重点考虑的则是各种符合环保要求的原料; 生产环节 又是考虑各种环保工艺、环保设备的运用, 等等。不同环节不同成本动因为我们利用作业成 本法的成本动因归集分配环境成本提供了基础。

具体核算账户的设计，企业可考虑设置 “环境成本”总账，再根据不同的成本动因设置 二级或三级明细账, 比如 “研究费用”、“排污费用” 等, 便于环境成本能够根据成本动因 归集与分配。值得注意的是, 企业发生的各种与产品生产直接相关的环境成本, 需计入企业 产品生产成本; 与产品生产无关的环境成本直接计入当期损益。也就是企业并非所有环境成 本均需计入 “环境成本”账户。

根据成本动因归集分配主要分为两类：一类是可直接归集与分配的，比如研发、工资等 环境成本，直接计入 “环境成本” ; 一类是需要通过一定方法进行分配的，比如各种环境污 染损失、自然资源损坏支出等, 由于其成本对象较为模糊, 需要借助一定技术手段进行归集 分配 ${ }^{[4]}$ 。

值得特别注意的是, 确认为产品成本的环境成本也是基于作业成本法进行的确认，根据 资源动因建立环境成本的作业成本库, 然后进一步利用作业动因再把环境成本归集分配到产 品的生产成本中。

\section{5. 结束语}


本文针对目前环境成本在确认、计量、披露及核算等各环节因缺少法律法规的指导而存 在的各种问题进行了剖析。在问题总结分析的基础上，笔者进一步的给出了相关的改善建 议。尤其针对环境成本核算方法的严重问题，笔者创新的利用作业成本法结合企业各个生产 环节，对现有的核算方法进行优化。通过该方法能够准确核算企业各个环节对应的环境成 本，明确各独立部门的成本责任，有利于企业环境成本控制。

当下环境成本会计处理方面的各种问题不利于企业环境责任的承担，更不利于 “环境友 好型的社会” 建设。环境成本会计不足带来的制度障碍也将对企业的效益提升及可持续发展 构成阻碍。正是这种制度的缺少, 目前大多数企业尚未意识环境成本会计的重要性, 导致企 业忽视环境责任只注重自身利益。两者陷入持续的恶性循环，这种局面急待打破。为此，一 方面急需政府建立健全各种与环境成本核算相关的会计制度和法律制度; 另一方面, 政府需 加强对企业各种环境成本核算财务人员的指导培养，也许适时提高企业的环保意识。只要做 到这两点, 才能使企业能够在战略高度重视环境成本核算, 遵守环境成本核算相关制度, 最 终有利于企业的可持续发展及国家 “环境友好型的社会”建设。

\section{References}

[1] Xiao Xu, Mao Hongtao Discussions on the Application of the Enterprise Environment Cost, Accounting Research, vol. 6, pp. 15-16,2010.

[2] Ministry of Finance of the People's Repubilc of China. Accounting Standard for Business Enterprises, Beijing Economic Science Press,pp. 122-124,2006.

[3] Pan Yushuang, Wei Wei Environment Cost Control of Manufacturing Enterprise under Value Chain Perspective, Finance and Accounting Monthly, vol. 16 , pp. 18-20,2013.

[4] Wang Liyan Environmental Cost Accounting and Environmental Accounting System Economic Science,vol. 6, pp. 35-36,1998. 